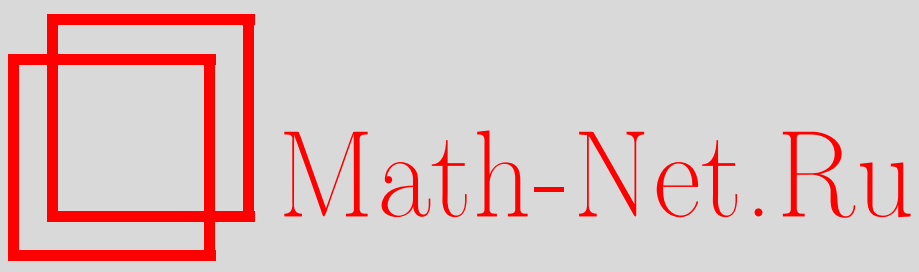

V. G. Romanov, A generalized gronwall-bellman inequality, Sibirsk. Mat. Zh., 2020, Volume 61, Number 3, 674-680

DOI: https://doi.org/10.33048/smzh.2020.61.315

Use of the all-Russian mathematical portal Math-Net.Ru implies that you have read and agreed to these terms of use http://www . mathnet.ru/eng/agreement

Download details:

IP: 18.234 .156 .22

April 26, 2023, 16:43:05

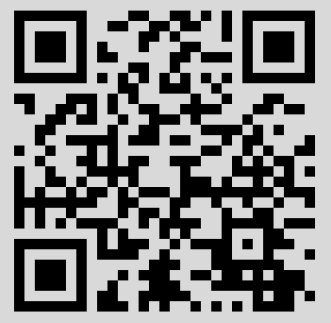


Сибирский математический журнал Май-июнь, 2020. Том 61, № 3

УДК 517.91

\title{
ОБОБЩЕННОЕ НЕРАВЕНСТВО ГРОНУОЛЛА — БЕЛЛМАНА
}

\section{В. Г. Романов}

\begin{abstract}
Аннотация. Рассматривается интегральное неравенство, в котором ядро представляет собой неотрицательный полином по степеням произведения разности аргументов и большого параметра $N$. Устанавливается неравенство, совпадающее по форме с известным неравенством Гронуолла - Беллмана, в котором аргумент экспоненты линейно зависит от $N$.
\end{abstract}

DOI 10.33048/smzh.2020.61.315

Ключевые слова: интегральное неравенство, лемма Гронуолла - Беллмана.

В теории дифференциальных уравнений часто встречается интегральное неравенство

$$
u(x) \leq A+\alpha \int_{0}^{x} u(\xi) d \xi, \quad x \in\left[0, x_{0}\right], x_{0}>0,
$$

в котором $A$ и $\alpha$ - некоторые неотрицательные постоянные, $u(x)$ - неотрицательная непрерывная на отрезке $\left[0, x_{0}\right]$ функция. Это неравенство часто возникает при оценке решений дифференциальных уравнений. Хорошо известна, так называемая лемма Гронуолла - Беллмана, которая дает оценку решения неравенства (1) (см. [1-3]).

Лемма Гронуолла - Беллмана. Для неотрицательной и непрерывной на $\left[0, x_{0}\right]$ функции $u(x)$, удовлетворяющей неравенству $(1)$, имеет место оценка

$$
u(x) \leq A e^{\alpha x}, \quad x \in\left[0, x_{0}\right] .
$$

Существуют многочисленные обобщения этой леммы. Пожалуй, наиболее общим является следующее утверждение, принадлежащее Х. Мовлянкулову и А. Н. Филатову (см. [4, теорема 1.4.1]).

Утверждение. Пусть непрерывная и неотрицательная на $\left[0, x_{0}\right]$ функция $u(x)$ удовлетворяет неравенству

$$
u(x) \leq a(x)+b(x) \int_{0}^{x} k(x, \xi) u(\xi) d \xi, \quad x \in\left[0, x_{0}\right]
$$

Работа выполнена при поддержке Математического центра в Академгородке, соглашение с Министерством науки и высшего образования Российской Федерации номер 075-15-20191613.

(c) 2020 Романов В. Г. 
в котором $a(x) \geq 0, b(x) \geq 0$ и $k(x, \xi) \geq 0$ - непрерывные функции при $0 \leq \xi \leq$ $x \leq x_{0}$. Тогда

$$
u(x) \leq A(x)+\exp \left(B(x) \int_{0}^{x} K(x, \xi) d \xi\right), \quad x \in\left[0, x_{0}\right] .
$$

В неравенстве (4)

$$
A(x)=\sup _{0 \leq \xi \leq x} a(\xi), \quad B(x)=\sup _{0 \leq \xi \leq x} b(\xi), \quad K(x, \xi)=\sup _{\xi \leq \sigma \leq x} k(\sigma, \xi) .
$$

Между тем, при оценке сходимости $N$ приближений решения некорректных задач встречаются интегральные неравенства, содержащие большой параметр $N$ (см., например, [5]). Эти неравенства имеют вид

$$
u(x) \leq A+N \int_{0}^{x} P_{m}(N(x-\xi)) u(\xi) d \xi, \quad x \in\left[0, x_{0}\right], x_{0}>0,
$$

в котором $A \geq 0, u(x) \geq 0$ - непрерывная на $\left[0, x_{0}\right]$ функция и $P_{m}(z)$ - полином степени $m \geq 1$,

$$
P_{m}(z)=\sum_{k=0}^{m} a_{k} \frac{z^{k}}{k !}
$$

с коэффициентами $a_{k} \geq 0$.

Параметр $N$ может принимать сколь угодно большие значения. При этом актуальна оценка функции $u(x)$, в которую этот параметр $N$ входит в экспоненту линейным образом. Насколько известно автору, в научной литературе подобного рода оценок нет. В частности, из приведенного выше утверждения, обобщающего лемму Гронуолла - Беллмана, следует оценка, в которой аргумент экспоненты зависит от $N$ полиномиально.

Для неравенства (6) оказывается справедливой

Теорема 1. Если непрерывная и неотрицательная на $\left[0, x_{0}\right]$ функция $u(x)$ удовлетворяет неравенству (6), то для нее справедлива оценка

$$
u(x) \leq A e^{\alpha x}, \quad x \in\left[0, x_{0}\right],
$$

в которой

$$
\alpha=N \max _{0 \leq k \leq m}\left(2^{m} a_{k} / C_{m}^{k}\right)^{1 /(k+1)} s
$$

и $C_{m}^{k}$ - биномиальные коэффициенты $C_{m}^{k}=m ! /(k !(m-k) !)$.

ДоказАтельство. При $m=0$ оценка (7) составляет суть неравенства Гронуолла - Беллмана. Поэтому в дальнейшем принимается, что $m \geq 1$. Из равенства (8) следует, что коэффициенты $a_{k}$ удовлетворяют неравенствам

$$
a_{k} N^{k+1} \leq 2^{-m} \alpha^{k+1} C_{m}^{k}, \quad k=\overline{0, m} .
$$

Усиливая неравенство (6), приходим к неравенству

$$
u(x) \leq A+\alpha \int_{0}^{x} Q_{m}(\alpha(x-\xi)) u(\xi) d \xi, \quad x \in\left[0, x_{0}\right],
$$


в котором полином $Q_{m}(z)$ определен формулой

$$
Q_{m}(z)=2^{-m} \sum_{k=0}^{m} C_{m}^{k} \frac{z^{k}}{k !} .
$$

Возникает естественный вопрос: чем неравенство (9) удобнее неравенства (6)? Отвлечемся на время от доказательства теоремы, чтобы дать ответ на этот вопрос. Вместо неравенства (9) рассмотрим соответствующее ему равенство

$$
y(x)=A+\alpha \int_{0}^{x} Q_{m}(\alpha(x-\xi)) y(\xi) d \xi, \quad x \in\left[0, x_{0}\right],
$$

для неотрицательной непрерывной функции $y(x)$. Нетрудно установить, что эта функция является решением следующей задачи Коши:

$$
\begin{gathered}
y^{(m+1)}(x)=2^{-m} \alpha\left(\frac{d}{d x}+\alpha\right)^{m} y(x), \quad x \in\left[0, x_{0}\right], \\
y(0)=A, \quad y^{(k)}(0)=2^{-m} A C_{m}^{k-1} \alpha^{k}, \quad k=\overline{1, m} .
\end{gathered}
$$

Характеристический полином, соответствующий дифференциальному уравнению (11), имеет вид

$$
\lambda^{m+1}(x)=2^{-m} \alpha(\lambda+\alpha)^{m} .
$$

Этот полином, очевидно, имеет вещественный корень $\lambda=\alpha$. Достаточно простой анализ показывает, что при $m$ четном $\lambda=\alpha$ является единственным вещественным корнем характеристического уравнения, остальные корни, если $m>1$, являются комплексно сопряженными. При $m$ нечетном существует еще один вещественный корень, принадлежащий интервалу $(-\alpha, 0)$, остальные корни, если $m>2$, также являются комплексно сопряженными. Чтобы оценить $y(x)$, надо найти (или по крайней мере оценить) все корни $\lambda_{s}$, $s=\overline{1, m+1}$, характеристического уравнения и затем выбрать линейную комбинацию $\sum_{s=1}^{m+1} c_{s} e^{\lambda_{s} x}$ так, чтобы удовлетворялись начальные данные в (11). Сделать это довольно сложно. Однако наличие у характеристического полинома действительного корня $\lambda=\alpha$ (по-видимому, имеющего наибольшую вещественную часть) позволяет сделать предположение, что $y(x) \leq A e^{\alpha x}$, которое эквивалентно утверждению теоремы 1 . Конечно, это лишь гипотеза, требующая доказательства. Фактически это доказательство приведено ниже. Мы пояснили только, почему неравенство (9) предпочтительнее неравенства (6).

Продолжим доказательство теоремы. Из (9) следуют очевидные неравенства

$$
\begin{aligned}
& u(x) \leq y_{0}(x)=A, \\
& u(x) \leq y_{n}(x)=A+\alpha \int_{0}^{x} Q_{m}(\alpha(x-\xi)) y_{n-1}(\xi) d \xi, \quad n=1, \ldots, x \in\left[0, x_{0}\right] .
\end{aligned}
$$

Введем в рассмотрение разности

$$
\tilde{y}_{n}(x)=y_{n}(x)-y_{n-1}(x), \quad n=1,2, \ldots .
$$

Из определения последовательности $y_{n}$ вытекают равенства

$$
\tilde{y}_{n}(x)=\alpha \int_{0}^{x} Q_{m}(\alpha(x-\xi)) \tilde{y}_{n-1}(\xi) d \xi, \quad n=1, \ldots, x \in\left[0, x_{0}\right] .
$$


Вычислим последовательно $\tilde{y}_{1}(x), \tilde{y}_{2}(x), \ldots, \tilde{y}_{n}(x)$. Из формул $(13)$ следует равенство

$$
\tilde{y}_{1}(x)=A \alpha \int_{0}^{x} Q_{m}(\alpha(x-\xi)) d \xi=2^{-m} A \sum_{k=0}^{m} C_{m}^{k} \frac{(\alpha x)^{k+1}}{(k+1) !}, \quad x \in\left[0, x_{0}\right] .
$$

Аналогично

$$
\begin{aligned}
& \tilde{y}_{2}(x)=A \alpha \int_{0}^{x} Q_{m}(\alpha(x-\xi)) \tilde{y}_{1}(\xi) d \xi \\
& \quad=2^{-2 m} A \sum_{k=0}^{m} \sum_{\ell=0}^{m} C_{m}^{k} C_{m}^{\ell} \alpha^{k+\ell+2} \int_{0}^{x} \frac{(x-\xi)^{k} \xi^{\ell+1}}{k !(\ell+1) !} d \xi, \quad x \in\left[0, x_{0}\right] .
\end{aligned}
$$

Используя интегрирование по частям, находим, что

$$
\int_{0}^{x} \frac{(x-\xi)^{k} \xi^{\ell+1}}{k !(\ell+1) !} d \xi=\frac{x^{k+\ell+2}}{(k+\ell+2) !}, \quad k, \ell=0,1, \ldots, m
$$

В силу этих равенств

$$
\tilde{y}_{2}(x)=2^{-2 m} A \sum_{k=0}^{m} \sum_{\ell=0}^{m} C_{m}^{k} C_{m}^{\ell} \frac{(\alpha x)^{k+\ell+2}}{(k+\ell+2) !}, \quad x \in\left[0, x_{0}\right] .
$$

Чтобы преобразовать двойную сумму, удобно доопределить совокупность чисел $C_{m}^{k}$ на все положительные целочисленные значения $k$, положив

$$
C_{m}^{k}=0, \quad k>m .
$$

Тогда равенство (17) можно представить в виде

$$
\begin{gathered}
\tilde{y}_{2}(x)=2^{-2 m} A \sum_{k=0}^{m} \sum_{\ell=0}^{\infty} C_{m}^{k} C_{m}^{\ell} \frac{(\alpha x)^{k+\ell+2}}{(k+\ell+2) !}=2^{-2 m} A \sum_{s=0}^{\infty} \sum_{k=0}^{s} C_{m}^{k} C_{m}^{s-k} \frac{(\alpha x)^{s+2}}{(s+2) !} \\
=2^{-2 m} A \sum_{s=0}^{\infty} C_{2 m}^{s} \frac{(\alpha x)^{s+2}}{(s+2) !}, \quad x \in\left[0, x_{0}\right] .
\end{gathered}
$$

Выше была использована формула свертки Вандермонда:

$$
C_{p+q}^{s}=\sum_{k=0}^{s} C_{p}^{k} C_{q}^{s-k}
$$

справедливая для любых целых неотрицательных $p, q$ и $s$.

Продолжая процесс вычислений, приходим к формуле

$$
\tilde{y}_{n}(x)=2^{-m n} A \sum_{s=0}^{\infty} C_{m n}^{s} \frac{(\alpha x)^{s+n}}{(s+n) !}, \quad n \geq 1, x \in\left[0, x_{0}\right] .
$$

Формула (20) уже установлена для $n=1$ и $n=2$ и легко обосновывается для любых $n$ методом математической индукции. Действительно, если примем, что 
она верна для $n=r-1, r \geq 3$, то в результате вычислений получаем следующую цепочку равенств:

$$
\begin{gathered}
\tilde{y}_{r}(x)=A \alpha \int_{0}^{x} Q_{m}(\alpha(x-\xi)) \tilde{y}_{r-1}(\xi) d \xi \\
=2^{-m r} A \sum_{k=0}^{m} \sum_{\ell=0}^{\infty} C_{m}^{k} C_{m(r-1)}^{\ell} \alpha^{k+\ell+r} \int_{0}^{x} \frac{(x-\xi)^{k} \xi^{\ell+r-1}}{k !(\ell+r-1) !} d \xi \\
=2^{-m r} A \sum_{k=0}^{m} \sum_{\ell=0}^{\infty} C_{m}^{k} C_{m(r-1)}^{\ell} \frac{(\alpha x)^{k+\ell+r}}{(k+\ell+r) !}=2^{-m r} A \sum_{s=0}^{\infty} \sum_{k=0}^{s} C_{m}^{k} C_{m(r-1)}^{s-k} \frac{(\alpha x)^{s+r}}{(s+r) !} \\
=2^{-m r} A \sum_{\ell=0}^{\infty} C_{m r}^{s} \frac{(\alpha x)^{s+r}}{(s+r) !}, \quad x \in\left[0, x_{0}\right] .
\end{gathered}
$$

В промежуточных выкладках использованы равенство (16) и формула (19) при $p=m$ и $q=m(r-1)$.

Таким образом, из предположения, что формула (20) верна для $n=r-1$, следует, что она верна и для $n=r$. Это оправдывает справедливость формулы (20) для любых $n$.

Рассмотрим ряд

$$
A+\sum_{n=1}^{\infty} \tilde{y}_{n}(x), \quad x \in\left[0, x_{0}\right]
$$

Подставляя в него вместо $\tilde{y}_{n}(x)$ правую часть формулы $(20)$, приходим к степенному ряду

$$
\begin{aligned}
A+\sum_{n=1}^{\infty} \tilde{y}_{n}(x) & =A+A \sum_{n=1}^{\infty} \sum_{\ell=0}^{\infty} 2^{-m n} C_{m n}^{\ell} \frac{(\alpha x)^{\ell+n}}{(\ell+n) !} \\
& =A \sum_{s=0}^{\infty} \frac{(\alpha x)^{s}}{s !} \sum_{n=1}^{\infty} 2^{-m n} C_{m n}^{s-n}=A \sum_{s=0}^{\infty} d_{s} \frac{(\alpha x)^{s}}{s !}, \quad x \in\left[0, x_{0}\right],
\end{aligned}
$$

в котором коэффициенты $d_{s}$ вычисляются по формуле

$$
d_{0}=1 ; \quad d_{s}=\sum_{n=1}^{s} 2^{-m n} C_{m n}^{s-n}, \quad s \geq 1 .
$$

Так как $2^{-m n} C_{m n}^{s-n}<1$ для $n \geq 1$, из формулы (23) следует (очень грубая) оценка $d_{s}<s$ для $s \geq 1$. Эта оценка тем не менее означает, что степенной ряд (22) равномерно сходится на отрезке $\left[0, x_{0}\right]$. Его сумма, очевидно, совпадает с предельной функцией последовательности $y_{n}(x)$, т. е. с функцией $y(x)=\lim _{n \rightarrow \infty} y_{n}(x)$, которая, в свою очередь, совпадает с решением интегрального уравнения (10), а значит, и с решением задачи Коши (11).

Ниже доказываем, что при любом $m \geq 1$ справедливы соотношения

$$
d_{s}<1, \quad s \geq 1 .
$$

Использовать для доказательства этого факта формулу (23) довольно затруднительно и мы предпочтем здесь иной путь. Воспользуемся тем, что функция $y(x)$ является решением уравнения (10). Следовательно,

$$
y(x)=A+A \alpha \int_{0}^{x} Q_{m}(\alpha(x-\xi)) \sum_{\ell=0}^{\infty} d_{\ell} \frac{(\alpha x)^{\ell}}{\ell !}
$$




$$
\begin{gathered}
=A+2^{-m} A \sum_{\ell=0}^{\infty} \sum_{k=0}^{m} C_{m}^{k} \alpha^{\ell+k+1} d_{\ell} \int_{0}^{x} \frac{(x-\xi)^{k} \xi^{\ell}}{k ! \ell !} d \xi \\
=A+2^{-m} A \sum_{\ell=0}^{\infty} \sum_{k=0}^{m} C_{m}^{k} d_{\ell} \frac{(\alpha x)^{\ell+k+1}}{(\ell+k+1) !} \\
=A+2^{-m} A \sum_{s=0}^{\infty} \frac{(\alpha x)^{s+1}}{(s+1) !} \sum_{k=0}^{s} C_{m}^{k} d_{s-k}, \quad x \in\left[0, x_{0}\right] .
\end{gathered}
$$

Из этого равенства получаем рекуррентную формулу

$$
d_{s+1}=2^{-m} \sum_{k=0}^{s} C_{m}^{k} d_{s-k}, s \geq 0 ; \quad d_{0}=1,
$$

из которой и вытекают неравенства (24). В самом деле, при $s=0$ находим, что $d_{1}=2^{-m} d_{0}<1$. Предположим теперь, что неравенства (24) выполнены для $s=\overline{1, r}, r \geq 2$. Тогда

$$
d_{r+1}=2^{-m} \sum_{k=0}^{r} C_{m}^{k} d_{r-k}<2^{-m} \sum_{k=0}^{r} C_{m}^{k} \leq 1 .
$$

Таким образом, методом математической индукции установлено, что неравенства (24) верны при любом $s \geq 1$. Отсюда следует, что справедливо неравенство

$$
u(x) \leq y(x)=A \sum_{s=0}^{\infty} d_{s} \frac{(\alpha x)^{s}}{s !} \leq A \sum_{s=0}^{\infty} \frac{(\alpha x)^{s}}{s !}=A e^{\alpha x}, \quad x \in\left[0, x_{0}\right],
$$

из которого и вытекает утверждение теоремы 1.

То обстоятельство, что все $d_{s}, s \geq 1$, строго меньше 1 , говорит о том, что оценка (7) не является оптимальной для неравенства (9). Ее можно улучшить, если найти точное решение задачи (11). В некоторых случаях это удается сделать (например, при $m=1$ и $m=2$ ). Чтобы не усложнять изложения, рассмотрим случай $m=1$. Для этого случая верна

Теорема 2. Если непрерывная и неотрицательная на $\left[0, x_{0}\right]$ функция $u(x)$ удовлетворяет неравенству (9) с $m=1$, то

$$
u(x) \leq A\left(\frac{2}{3} e^{\alpha x}+\frac{1}{3} e^{-\alpha x / 2}\right), \quad x \in\left[0, x_{0}\right] .
$$

ДокАЗАТЕЛЬСтво. Непосредственно проверяется, что при $m=1$ решение задачи (11) имеет вид

$$
y(x)=A\left(\frac{2}{3} e^{\alpha x}+\frac{1}{3} e^{-\alpha x / 2}\right), \quad x \in\left[0, x_{0}\right] .
$$

Отсюда следует оценка (26). 


\section{ЛИТЕРАТУРА}

1. Беллман $P$. Теория устойчивости решений дифференциальных уравнений. М.: Изд-во иностр. лит., 1954.

2. Беккенбах Э., Беллман Р. Неравенства. М.: Мир, 1965.

3. Демидович Б. П. Лекции по математической теории устойчивости. М.: Наука, 1967.

4. Филатов А. Н., Шарова Л. В. Интегральные неравенства и теория нелинейных колебаний. М.: Наука, 1976.

5. Романов В. Г. Регуляризация задачи Коши с данными на времени-подобной плоскости // Сиб. мат. журн. 2018. Т. 59, № 4. С. 879-890.

Поступила в редакиию 5 марта 2020 2.

После доработки 5 марта 2020 г.

Принята к публикации 8 апреля 2020 г.

Романов Владимир Гаврилович

Институт математики им. С. Л. Соболева СО РАН,

пр. Академика Коптюга, 4, Новосибирск 630090

romanov@math.nsc.ru 